\title{
Application Research on the analysis of Biological detection image segmentation using PDE
}

\author{
Wang Baozhen, Chen Min \\ Department of Mathematics, Huanghuai University, Zhumadian, 463000, China \\ Department of Mathematics, Huanghuai University, Zhumadian, 463000, China
}

Keywords: Partial differential equation; biological detection; image segmentation; application model

\begin{abstract}
Partial differential equation(PDE) plays an important role in the field of image segmentation in biological detection. According to the analysis of some problems existing in the process of current biological detection image segmentation, this research set up a kind of image analysis and processing of biological detection algorithm based on PDE.Through the analysis of PDE in image processing and application situation of development methods, this study constructed a biological detection image segmentation application model based on PDE. The results show that the PDE is a mathematical operation as the foundation, and with an open framework for development mode. PDE is a biological detection very effective image segmentation analysis tool that can solve the image topology in biology of high complexity and noise problems.
\end{abstract}

\section{Introduction}

Image segmentation refers to the known image of the original split into several sub region has some special properties, and will be of interest to target separation process. Image segmentation in the practical application of the process is a necessary image processing technology, and has been widely concerned by the field of biology and medical science field, played a very important role in image processing and analysis process. Image segmentation is not only affect the feature extraction, the premise condition is also the expression of target expression, feature extraction based on image segmentation and target, can be the initial image abstraction and compact, contribute to the realization of the image of a higher level of understanding and analysis of desire. At present, the relevant image segmentation analysis technology research, has acquired a wealth of theoretical analysis results and research methods, concrete can be divided into the following four categories: region based segmentation segmentation method, use threshold based analysis method, in particular theoretical tool based segmentation and edge based analysis method segmentation analysis method. And the image analysis method by PDEs is a main branch of system analysis of image segmentation field, it has very important value in the entire image analysis and processing process.

Because of the topological complexity, noise, high inter individual difference and contrast characteristics, unified description of biological image is not easy.At the same time, the target object with deformable features, plus the accompanying non rigid motion, resulting in many other fields in the area of success are not can be directly applied to the segmentation of image analysis in biological detection.PDE on mathematics deep foundation and processing frame flexible frame, effective integration of some essential theoretical, can satisfy the need for different application scenarios and different imaging mode, has gradually become one of the most popular tools for image in the field of biological detection.PDE in the contour model, nonlinear diffusion model and other methods, and biological testing of image segmentation are widely applied in the analysis of.In order to achieve better biological detection image visualization and maneuverability, select repeat degree is high, precision, high efficiency and accurate positioning method of PDE, to biological images for quantitative detection.

\section{Mathematical connotation of PDE}

If a certain differential equations appear only contains unknown function has an argument, then it 
can be called the differential equations of ordinary differential equations, but also means that the differential equations of general conditions.If a differential equation exists in multiple partial derivative, or say if the unknown functions with multiple variables and equations of correlation exists, there will be derivative and unknown functions of several variables corresponding to each other, then it can be called the differential equation of PDE.

At present, the construction of many fields in the equation not only contains the partial derivative process on time, but also contains the process of seeking the partial derivatives of the spatial variables, this equation can also be referred to as PDE or a combination of PDE.Compared to the classical PDE include the activities of the partial differential equation (hyperbolic partial differential equation),Poisson's partial differential equation (elliptic partial differential equations), the heat conduction type partial differential equation (parabolic partial differential equations), in particular to:

1. $\quad$ EPDE: $\frac{\partial^{2} u}{\partial x^{2}}+\frac{\partial^{2} u}{\partial y^{2}}=f(x, y)$

2. PPDE: $\frac{\partial u}{\partial t}=\frac{\partial^{2} u}{\partial x^{2}}+\frac{\partial^{2} u}{\partial y^{2}}$

3. HPDE: $\frac{\partial^{2} u}{\partial t^{2}}=\frac{\partial^{2} u}{\partial x^{2}}$

PDE only in the appropriate initial and boundary conditions known conditions can be solved, is also specified conditions should be reasonable, which can be converted into a fixed solution problem.If the type of heat conduction PDE requirements (PDE of parabolic type) definite solution, the specific method for:

$$
\begin{aligned}
& \frac{\partial u}{\partial t}=\lambda \Delta u, \quad x \in \Omega \\
& u(x, 0)=u_{0}(x), \quad x \in \Omega \\
& u(x, t)=f(x), \quad x \in \partial \Omega \\
& u(x, 0)=u_{0}(x) \text { is the initial conditions for PDE, } u(x, t)=f(x) \text { is for PDE with boundary }
\end{aligned}
$$

conditions.If in a specific region, the solution of a partial differential equation with existence and uniqueness, stability, and can be called posed, on the contrary, it is ill posed problem.

Process for solving the PDE of the boundary condition in the following three categories:

The first category: $u(x, t)=f(x), x \in \partial \Omega, t \geq 0$, is the Dirichlet boundary condition.

The second category: $\frac{\partial u}{\partial \eta}=f(x, t), x \in \partial \Omega, \eta$ is the vector of $\partial \Omega$, and this boundary can be called the Neumann boundary condition.

The third category: $\frac{\partial u}{\partial \eta}+\lambda u=f(x, t), x \in \partial \Omega$, is the Robin boundary conditions.

\section{Analysis of Biological detection image segmentation using PDE}

In the course of the study image segmentation analysis application, some foreign researchers have found that the active contour model, and points out that this model can realize the analysis of biological image segmentation for solving PDE under the condition of.The core of the guiding ideology of the active contour model is in a surface or a curve defined on a deformation of the energy function, the main composition of the deformation energy function is external energy $\left(E_{\text {out }}\right)$ and internal energy $\left(E_{\text {in }}\right)$.The external energy $\left(E_{\text {out }}\right)$ refer to the power in the active surface, is based on the characteristics of biological image self extraction as the basis, the formation of external force field on a to deformation energy constraints in the regional characteristics of biological image.The 
internal energy $\left(E_{\text {in }}\right)$ refer to the main control points on the curvature degree and continuity of each point, it is mainly used to describe the deformation state of a surface with a curve is elastic or distorted.Therefore, the change status of the active contour model under different stress effects many other control, these forces will generate part of the energy, at the same time, the energy can be used to represent the active contour model relative independence of energy.

Active contour model is applied to the analysis of biological process in the field of image segmentation, is the reference position and shape and the location of contours in the active contour model control of many energy expansion evolution activity, and eventually find out the dynamic process of minimizing the energy position.Therefore, looking for the energy minimization problem is not static, but a dynamic problem.At the same time evolution of active contour model also may be affected by the law of elasticity and restriction of Lagrange law of dynamics, and in numerous force joint utility under the condition that the energy surface is a continuous evolution, until all of the effectiveness are in balance state stopped.The balance force of the state can be thought of as the minimization of energy function of the state, therefore, the segmentation problem analysis of biological images can be converted to find a solution of the optimal energy function of active contour model.

In the solving process of active contour model, also contains the achievements of research on computer vision theory deeply the 。In recent years, the theory circle and the practice circle based on active contour model, explore a lot better segmentation effect analysis, the wider adaptation model.For example, in domestic academic scholars have in regional perspective improved level set image segmentation method and innovation improved geodesic active contour model and so on, these methods for some complicated graphics features of feasibility analysis to find the segmentation, and then promote the domestic image segmentation analysis enhance adaptive capacity, reduce the evolution the time limit for PDE, in the perspective of biological image segmentation, provides the theoretical basis for the analysis research.

\section{Application model of analysis of Biological detection image segmentation using PDE}

PDE in the basic concept of biological detection image segmentation application model construction analysis is, reference features of biological images needs, construct a partial differential equation model about biological image energy, the partial differential model solution is also the demand of biological detection of image edge features.PDE is an essential method, analysis of the current biological image segmentation, active contour model is the main model of partial differential equations in biological detection image segmentation analysis application.

Active contour model is mainly refers to that defined in biological image region in the curve, at the same time, the outer edge of the curve is jointly dominated in the internal and external force gradually trend of biological images, and toward the biological image edge motion driven by external force active contour, the internal force is persistent to maintain active contours and smooth, and in the total energy minimization model to converge to the biological image edge.The theory of active contour model of rapid development of activity model is mainly attributed to the parameters, and the relevant analysis of the parametric active contour model led also to the emergence of the geometric active contour model.In the biological field of image segmentation, compared with other image segmentation methods of analysis, image segmentation is a partial differential equation analysis method based on the advantages of extraction process of constraint conditions of biological images are mainly concentrated in the same image characteristics, after the initial change, can converge in the energy minimum state, also has adaptability and strong robustness.Because the curve presents the way differences, active contour model which also can have two kinds, namely geometric active model and parameters for activity model.

Parametric active biological detection image segmentation model. Parametric active biological model detection image segmentation is also the parameter active contour model for image segmentation of biological detection, mainly by the 2D curve a variability of the composition, the two-dimensional curve of this variable in the process of finding the edge of the continuous 
deformation occurred, finally converge to the given target position. Which can be expressed by a mathematical formula:

Suppose, there are $u(x)$ contains biological image function boundary, and $u(x)$ defined in the $\Omega \subset R^{2} \rightarrow R$ range, $g(v)=[x(v), y(v)]$ refers to the plane curve parametric conditions. So the existence of the following energy function in biological image area:

$$
\begin{aligned}
E[g(v)] & =E_{\text {in }}[g(v)]+E_{\text {out }}[g(v)]= \\
& \int_{0}^{1} \frac{1}{2}\left[a\left|g^{\prime}(v)\right|^{2}+b\left|g^{\prime \prime}(v)\right|^{2}\right] d v \\
& +\int_{0}^{1} h\{u[g(v)]\} d v
\end{aligned}
$$

$E_{\text {in }}[g(v)]$ is the internal energy,can control the smoothness of the curve. $E_{\text {out }}[g(v)]$ is the external energy, mainly used to attract the active contour of object boundaries.Among them, a, $b$ is to adjust the related parameters, $h[u(x)]$ is a potential function, mainly used to detect the edge feature of image,the range is $-\left\lfloor\nabla u(x)^{2}\right\rfloor$.After determining the adjustment parameters, segmentation problem analysis translates into finding the contour $g(v)$, so that $E[g(v)]$ is minimized, and then converted to determine the optimal solution of the problem.

In the variational method, the functional extreme value problem can be transformed into the solution of the problem for PDE.The conversion of the above models according to different application conditions, can appear in the partial differential equations of different types, such as Peron P and Malik J equation formula (2) with Osher S and Rudin as shown in equation formula:

$$
\left\{\begin{array}{l}
\frac{\partial u}{\partial t}=-\operatorname{div}[f(D u) D u] \\
u(0, x)=h(x)
\end{array}\right.
$$

But in the actual application process, structure parameter active contour biological detection segmentation model has a topological curve change is not obvious in the segmentation process.Therefore, in the original analysis model, biological image object are needed to advance the definition of a curve around the initialize it, only in this way can be divided obviously results.

Geometric active biological detection image segmentation model. Geometric active model of biological detection image segmentation is the segmentation of geometric active contour model of biological detection image, this model mainly through changes in normal vector and curve rate and related parameters to limit curve, at the same time, it also can convert the plane curve closed for the same curve and the value of $z=f(x, y)$ function, under normal circumstances, choose $\{z \mid z=0\}$ said the zero set, $f(x, y)$ is used to represent the function horizontal condition.Biological detection image curve in the level set segmentation in the context, purpose is not to track the curve position after the segmentation, mainly is the update function, and eventually realize the function of implicit zero level set segmentation purpose.The general is a simple function of level set function, mainly in order to compute the image segmentation curve topology processing biological detection is convenient and can be better, as shown in figure 1:

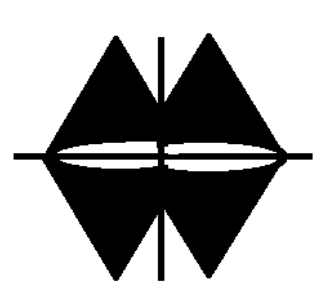

(a)

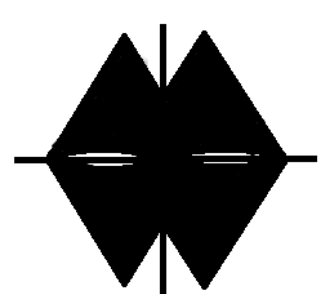

(b)

Figure 1: schematic diagram of segmentation level biological detection 
Pictured above, (a) is a biological curve is a closed curve in biology, after a period of time after the split into two closed (b).Thus it can be seen that the geometric active contour segmentation in biological monitoring image model results is quite obvious, that it can well solve the problem of biological monitoring image segmentation.

\section{Conclusions}

With the help of the partial differential equation to analyze biological detection image segmentation, can be biological image processing more standardized, stronger applicability.In this paper, the PDEs perspective, analysis of the segmentation of biological detection image analysis problems, through the mathematical analysis of the connotation of the PDE, discusses the PDE in the segmentation application in analysis of biological images, and construct a PDE in the segmentation for the analysis of biological detection image, enhance the stability of biological detection image segmentation and accuracy, it has certain practical value.

\section{References}

[1] Hu C,Jiang LJ,Bo J.Wavelet Transform and Morphology Image Segmentation Algorism for Blood Cell. 4th IEEE Conference on Industrial Electronics and Applications . 2009

[2] Gal Y,Mehnert A J H,Bradley A P,McMahon K,Kennedy D,Crozier S.Denoising of dynamic contrast-enhanced MR images using dynamic nonlocal means. IEEE Transactions on Medical Imaging . 2010

[3] $\mathrm{Li} \mathrm{C,Xu} \mathrm{C,Gui} \mathrm{C,et} \mathrm{al.Distance} \mathrm{regularized} \mathrm{level} \mathrm{set} \mathrm{evolution} \mathrm{and} \mathrm{its} \mathrm{application} \mathrm{to} \mathrm{image}$ segmentation. IEEE Transactions on Image Processing . 2010

[4] Ceylan, M,Ozbay, Y,Yildirim, E.A new approach for biomedical image segmentation:Combined complex-valued artificial neural network case study:Lung segmentation on chest CT images. 5th Cairo International Biomedical Engineering Conference . 2010

[5] Pouya Dehghani Tafti,Ricard Delgado-Gonzalo,Aurelien F Stalder,Michael Unser.Variational enhancement and denosing of flow field images. The eighth IEEE International Symposium on Biomedical Imaging . 2011

[6] M.A.Mohamed,B.M.EI-Den.Implementation of image fusion techniques using FPGA. IJCSNS International Journal of Computer Science and Network Security . 2010

[7] PJin LI,P PYanwei WANG,P P Jie WEI,P Dada WANG,PHong YU.A FNL Means De-noise Algorithm Based on Gradient Calibration. International Journal of Advancements in Computing Technology . 2011 